\title{
A Novel Adaptive Control Method for SVC Based on I\&I Stabilization and Adaptive Sliding-mode Observer
}

\author{
${ }^{1}$ Lei Zhang*, ${ }^{1}$ Junfeng Jing and ${ }^{1}$ Xiaohua Wang \\ (College of Electronics and Information, Xi 'an Polytechnic University, Xi 'an \\ 710048, P.R. China) \\ carol1208@163.com
}

\begin{abstract}
This paper presents the transient stability enhancement of power transmission system with Static Var Compensator (SVC) by adopting immersion and invariant (I\&I) methodology in designing SVC adaptive controller. The controlled system is a single machine infinite bus (SMIB) system with uncertain parameters: damping coefficient, mechanical power, generator rotor angle and line impedance. In the proposed SVC controller, a deduced-order target system is first defined and the dynamics of the controlled system will be "immersed" into it based on I\&I stabilization. The proposed SVC controller is then made adaptive by designing adaptive update laws based on adaptive sliding-mode observer to identify the uncertain parameters. The simulation results demonstrate the effectiveness of the proposed SVC adaptive controller.
\end{abstract}

Keywords: Immersion and Invariant (I\&I) Stabilization; Adaptive Sliding-mode Observer; Transient Stability; Static Var Compensator (SVC)

\section{Introduction}

In the present power transmission system, transient stability becomes one of the most significant and urgent issues because of explosive incremental of power requirements. Meanwhile, huge cost of building new transmission lines hinders constructing new lines massively. How to effectively enhance the transmitted power mostly depends on the transient stability margin of the transmission system [1]. Recently, with the development of power electronics technology, flexible AC transmission system (FACTS) devices are commonly used to improve the transient stability, increase the transmitted power and extend the transient stability margin of power transmission system [2]. Static Var Compensator (SVC) is the most commonly used FACTS device in practical engineering. The SVC controller changes the equivalent susceptance of the device by controlling the firing angle of thyristor-controlled reactor (TCR), which is the kernel component of SVC. By this way, SVC can provide dynamic voltage support and damp oscillations in power transmission system. Therefore designing an effective SVC controller is the most essential issue in improving transient stability of power transmission system.

The traditional linear control methods such as linear PID, inverse system are based on the approximately linearized system model on some operation equilibrium. In these cases the nonlinear characters of the power system and the changing operating conditions are not taken into account.

However, the problem of losing transient stability is related with some issues [3-4]. First, although the differential geometry based control methods, such as feedback linearization and nonlinear optimization, use the nonlinear system model, they still need to linearize the original model, and require exactly known parameters and invariable operating conditions [5-7]. Therefore, these kinds of control methods cannot guarantee the system's transient stability because they cannot identify the uncertain parameters and they cannot work 
effectively when the operating conditions changed considerably. Nowadays, adaptive backstepping, which is a recursive systematic algorithm, has been proven can improve the transient performance of nonlinear systems [8-10]. However, the control law designing of these methods are based on certainty-equivalence principle and the candidate Lyapunov function (CLF) must be constructive. In order to avoid constructing CLFs and coming out "computation inflation" phenomenon, an alternative scheme to adaptive backstepping, which does not follow the classical certainty-equivalence principle is (system) immersion and (manifold) invariance (I\&I) methodology and is presented in [12-13]. The basic idea of I\&I stabilization is to achieve the control objective by immersing the dynamics of the controlled system into a (possibly lower-order) target system that possesses the desired dynamic performance. It differs from most of the existing adaptive methods because it does not need to construct CLF. It has been applied in excitation controller design to improve the transient stability in power system [14]. Its application in improving transient stability of multi-machine power system is presented in [15]. In most existing nonlinear SVC controllers, the generator rotor angle, line impedance, damping coefficient and mechanical power of the controlled system are seemed as measurable parameters. And in many previous studies, only the damping coefficient of the system is considered as an uncertain parameter $[10,16]$. However, the four parameters mentioned above are practically cannot be measured, and the neglecting of the parametric uncertainties has adverse effects on the system transient performance. Aiming at this problem, two equivalent equations of substitutions of measurable parameters for the uncertain generator rotor angle and line impedance are presented in [17]. Besides, the identifications of the rest unmeasurable parameters should depend on adaptive control methods. Adaptive sliding-mode observer is presented in [18] for the first time, it can provide robust identifications for time-varying uncertain parameters. And then this method is improved and applied to induction motors

In this paper, the transient stability improvement of a SMIB system with proposed SVC controller is addressed, four unmeasurable parameters mentioned above are taken into account simultaneously. The generator rotor angle and line impedance are expressed by measurable parameters of the power system, and the damping coefficient and mechanical power are identified by designing adaptive update laws based on adaptive sliding-mode observer. The SVC controller design is based on I\&I stabilization, a target system is defined and then the controlled system is immersed into it. The simulation result shows that the transient stability of the controlled system is improved when a severe fault occurs by using the proposed SVC adaptive controller, and the proposed adaptive laws can identify the uncertain parameters when they changed largely.

\section{System Model and Problem Formulation Procedure}

\subsection{SMIB System with SVC}

In this paper, the researched model is a SMIB power system with SVC equipped in the middle of the transmission line. The configuration of the controlled system is depicted in Figure 1. The dynamics of SVC is a first-order inertia and its equivalent transfer function is depicted in Figure 2. 


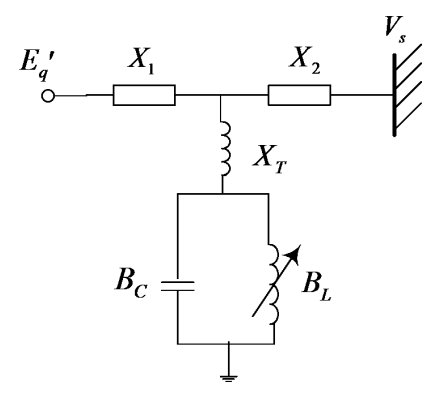

Figure 1. Single Machine Infinite Bus (SMIB) System with SVC

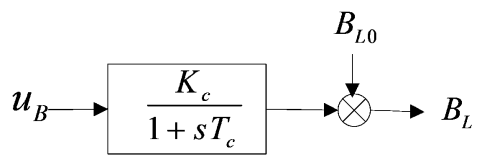

\section{Figure 2. Equivalent Transfer Function of SVC}

The mathematical description of the SMIB system with SVC controller can be depicted as the following differential equalities:

$$
\left\{\begin{array}{l}
\dot{\delta}=\omega-\omega_{0} \\
\dot{\omega}=\frac{\omega_{0}}{H}\left(P_{m}-P_{e}\right)-\frac{D}{H}\left(\omega-\omega_{0}\right) \\
\dot{B}_{S V C}=\frac{1}{T_{c}}\left(-B_{S V C}+B_{S V C 0}+K_{c} u_{B}\right)
\end{array}\right.
$$

where $\delta$ is the generator rotor angle; $\omega$ is the generator rotor angular speed; $\omega_{0}$ is the rating value of $\omega ; H$ is the inertia constant; $P_{m}$ is the mechanical power; $D$ is the unit damping coefficient; $P_{e}=E_{q}^{\prime} V_{s} B_{S V C} \sin \delta$ is the generator electrical power; $E_{q}{ }^{\prime}$ is the transient electromotive force of the quadrature axis of the generator; $V_{s}$ is the infinite bus voltage; $B_{S V C}=1 / X_{1}+X_{2}-X_{1} X_{2}\left(B_{L}-B_{C}\right)$ is the equivalent susceptance of the SVC system; $B_{C}$ is the susceptance of the capacitor of the SVC; $B_{S V C 0}$ is the initial steady-state value of $B_{S V C} ; B_{L}$ is the instantaneous equivalent susceptance of the connected inductor of the SVC; $B_{L}{ }^{\prime}$ is the total susceptance of the inductor of the SVC; $X_{1}=x_{d}{ }^{\prime}+X_{T}+X_{L 1}$ is the total impedance from the generator to the injection of the SVC device; $X_{2}=X_{L 2}$ is the total impedance from the injection of the SVC device to the infinite bus; $x_{d}{ }^{\prime}$ is the transient reactance of the direct axis of the generator; $X_{T}$ is the reactance of the transformer; $X_{L}$ is half of the transmission line's reactance and $X_{L 1}=X_{L 2}=X_{L} ; T_{c}$ is the time constant of the SVC controller; $K_{c}$ is the gain of the SVC controller; $u_{B}$ is the equivalence input of the SVC controller.

Then we definite new states and parameters to simplify the system (1):

$$
\begin{gathered}
x_{1}=\delta-\delta_{0} ; x_{2}=\omega-\omega_{0} ; x_{3}=B_{S V C}-B_{S V C 0} ; k_{1}=\omega_{0} / H ; k_{2}=\omega_{0} E_{q}^{\prime} V_{s} / H ; \\
k_{3}=1 / T_{c} ; \theta_{1}=-D / H ; \theta_{2}=P_{m} ; \delta_{\text {sin }}=\sin \left(\delta_{0}+x_{1}\right) ; \delta_{\text {cos }}=\cos \left(\delta_{0}+x_{1}\right) .
\end{gathered}
$$

By doing these, the system model (1) can be manipulated as: 


$$
\left\{\begin{array}{l}
\dot{x}_{1}=x_{2} \\
\dot{x}_{2}=\theta_{1} x_{2}+k_{1} \theta_{2}-k_{2}\left(x_{3}+B_{S V C 0}\right) \delta_{\mathrm{sin}} \\
\dot{x}_{3}=k_{3}\left(-x_{3}+K_{c} u_{B}\right)
\end{array}\right.
$$

The initial stable operation equilibrium point is $\left[\begin{array}{lll}\delta_{0} & \omega_{0} & B_{S V C 0}\end{array}\right]^{T}$. The state $x=\operatorname{col}\left(x_{1}, x_{2}, x_{3}\right) \in \ell$ denotes the difference between current operating conditions and the initial equilibrium. The three states represent the differences of rotor angle, rotor angular speed and the equivalent susceptance of SVC, respectively. The set $\ell=\left\{\delta-\delta_{0}, \omega-\omega_{0}, B_{S V C}-B_{S V C O} \mid 0<\delta<\pi / 2\right\}$ defines the region of the operating conditions.

Remark 1: In model (1), the generator rotor angle $\delta$, mechanical power $P_{m}$, unit damping coefficient $D$ and line impedance $X_{L}$ are cannot be measured practically, which blocks the applications of nonlinear control methods in power system. And the neglecting of the uncertainties has adverse effects on the system transient performance.

We make some parameter substitutions in order to solve this practical unmeasurable problem [17]:

Let $x_{s}=X_{T}+X_{L}$ and it is redefined as:

$$
x_{s}=\frac{-Q V_{s}^{2} \pm \sqrt{Q^{2} V_{s}^{2}-\left(Q^{2}+P_{e}^{2}\right) V_{s}^{2}\left(V_{s}^{2}-V_{t}^{2}\right)}}{Q^{2}+P_{e}^{2}}
$$

and

$$
d=\operatorname{arccot}\left(\frac{V_{s}}{x_{s} P_{e}}\left(-\frac{x_{d} V_{s}}{X_{1}}+\sqrt{V_{t}^{2}-\frac{x_{s}^{2}}{V_{s}^{2}} P_{e}^{2}}\right)\right)
$$

where $V_{t}$ is the terminal voltage of the generator; $Q$ is inactive power.

\subsection{Control Objective}

Firstly, the new stabilized operation equilibrium is denoted by $x^{*}=\operatorname{col}\left(x_{1}{ }^{*}, 0, x_{3}{ }^{*}\right)$ in the set $\ell$.

The control objective of the proposed SVC controller is concluded as follows:

Find a control law $u_{B}$ such that the response trajectories of the system states satisfy

$$
x(t) \in \ell, \forall t \geq 0 \Rightarrow \lim _{t \rightarrow \infty} x(t)=x^{*}
$$

The control objective means all the trajectories of the system (2) are globally bounded and will converge to the new stable equilibrium $x^{*}$. That is to say the new equilibrium $x^{*}$ is an attractive point for all trajectories that started from the set $\ell$.

\section{SVC Controller Design based on I \& I Stabilization}

\subsection{Overview of I\&I Method}

In this section, we state the brief results of I\&I stabilization, which is the foundation of the proposed SVC controller. For the globally asymptotically stabilization control of nonlinear systems, the basic result of I\&I stabilization is a set of sufficient conditions.

Theorem 1: Consider the following first-order affine nonlinear system

$$
\dot{x}=f(x)+g(x) u
$$

with $x \in R^{n}, u \in R^{m}$ and $x^{*} \in R^{n}$ denotes a certain equilibrium point to be stabilized. 
Assume that there exist the following smooth mappings: $\alpha: R^{p} \rightarrow R^{p} ; \pi: R^{p} \rightarrow R^{n}$; $\phi: R^{n} \rightarrow R^{n-p} ; c: R^{p} \rightarrow R^{m}$ and $\psi: R^{n \times(n-p)} \rightarrow R^{m}$ with $p<n$, such that the following (C1)-(C4) are completed.

(C1) Target system. There exists a mapping

$$
\dot{\xi}=\alpha(\xi)
$$

with state $\xi \in R^{p}$, has a globally asymptotically stable equilibrium at $\xi_{*} \in R^{p}$, with the corresponding mapping $x^{*}=\pi\left(\xi_{*}\right)$.

(C2) Immersion condition. For all $\xi \in R^{P}$

$$
f(\pi(\xi))+g(\pi(\xi)) x(\pi(\xi))=\frac{\partial \pi}{\partial \xi} \alpha(\xi)
$$

(C3) Implicit manifold. The following set identity holds

$$
\left\{x \in R^{n} \mid \varphi(x)=0\right\}=\left\{x \in R^{n} \mid x=\pi(\xi), \quad \xi \in R^{p}\right\}
$$

(C4) Manifold attractively and trajectory boundness. All trajectories of the system consist of (10) and (11):

$$
\begin{gathered}
\dot{z}=\frac{\partial \varphi}{\partial x}[f(x)+g(x) \psi(x, z)] \\
\dot{x}=f(x)+g(x) \psi(x, z)
\end{gathered}
$$

are bounded and satisfy

$$
\lim _{t \rightarrow \infty} z(t)=0
$$

where $z(t)=\varphi(x)$ and $u=\psi(x, z)$.

Then $x^{*}$ is a globally asymptotically stable equilibrium point of the following closedloop system (13):

$$
\dot{x}=f(x)+g(x) \psi(x, \varphi(x))
$$

Then we can summarize that the three pivotal steps for the I\&I stabilization control design are:

Step1: Define a target system which has a globally asymptotically stable equilibrium point and the degree of the target system strictly lower than the controlled system;

Step2: Construct a manifold $\mathcal{M}$, described implicitly by $\left\{x \in R^{n} \mid \varphi(x)=0\right\}$, and in parameterized form by $\left\{x \in R^{n} \mid x=\pi(\xi), \xi \in R^{P}\right\}$, which can be rendered invariant and attractive through designing a control law. And such that the restriction of the closed-loop system (13) to $\mathcal{M}$ is described by (7);

Step3: Design the "intermediate control law" $u=v(\pi(\xi))$ to make the manifold $\mathcal{M}$ invariant. A measurement of the distance of the system trajectories to the manifold $\mathcal{M}$ is expressed by $z$, which is so called "off-the-manifold" coordinate. Then the control objective is to design the "actual control law" $u_{B}=\psi(x, z)$ that drive $z$ to zero, keep the manifold $\mathcal{M}$ attractive, and guarantee the system trajectories bounded simultaneously.

\subsection{SVC Controller Design}

In this section the SVC controller is designed for the controlled system (1) using I\&I stabilization. We proceed to verify the (C1)-(C4) of Theorem 1 sequentially.

(C1) Target System. The pivotal step is to select a two-dimensional target system, into which the three-dimensional controlled system will be immersed. Here we select the dynamics of a pendulum as the target system, whose dynamics is presented as: 


$$
\sum_{T}:\left\{\begin{array}{l}
\dot{\xi}_{1}=\xi_{2} \\
\dot{\xi}_{2}=-V^{\prime}\left(\xi_{1}\right)-R\left(\xi_{1}, \xi_{2}\right) \xi_{2}
\end{array}\right.
$$

where $V\left(\xi_{1}\right)$ denotes the potential energy of the target system. $R\left(\xi_{1}, \xi_{2}\right)$ is the damping function. These two functions will be chosen in the consequent design. Assume that the system (14) has a globally asymptotically stable equilibrium point at $\xi_{*}=\left(\xi_{1^{*}}, 0\right)$ with the energy function is selected as:

$$
H(\xi)=\frac{1}{2} \xi_{2}^{2}+V\left(\xi_{1}\right)
$$

To ensure the stability of the closed-loop system (14) at the equilibrium $\xi_{*}$, the following assumption is made

\section{Assumption 1:}

A1) The potential energy function $V\left(\xi_{1}\right)$ satisfies $V^{\prime}\left(\xi_{1^{*}}\right)=0$ and $V^{\prime \prime}\left(\xi_{1^{*}}\right)>0$.

A2) The selection of the damping function satisfies $R\left(\xi_{1^{*}}, \xi_{2^{*}}\right)>0$.

(C2) Immersion condition. By Theorem 1, the mapping $\pi: R^{p} \times R^{n} \in \ell$ can transfer the coordinate frame of the controlled system to that of the target system. We defined $\pi$ as

$$
\pi(\xi)=\left(\xi_{1}, \xi_{2}, \pi_{3}(\xi)\right)
$$

where $\pi_{3}(\xi)$ is a function of state $\xi_{1}$ and it will be defined in the subsequent deducing. Then based on the immersion condition (8), the mapping $\pi$ and the controlled system dynamics are introduced, it can be got:

$$
\begin{aligned}
& \theta_{1} \xi_{2}+k_{1} \theta_{2}-k_{2}\left(\pi_{3}+B_{S V C 0}\right) \sin \left(\xi_{1}+\delta_{0}\right)=-R\left(\xi_{1}, \xi_{2}\right) \xi_{2}-V^{\prime}\left(\xi_{1}\right) \\
& k_{3}\left(-\pi_{3}+K_{c} v(\pi(\xi))=\frac{\partial \pi_{3}}{\partial \xi_{1}} \xi_{2}+\frac{\partial \pi_{3}}{\partial \xi_{2}}\left(-R\left(\xi_{1}, \xi_{2}\right) \xi_{2}-V^{\prime}\left(\xi_{1}\right)\right)\right.
\end{aligned}
$$

From (17), we choose $R\left(\xi_{1}, \xi_{2}\right)=-\theta_{1}>0$, so Assumption (A2) is satisfied. And we let $V\left(\xi_{1}\right)=-\beta \cos (\tilde{\xi})$ where $\beta>0$ will be chosen and $\tilde{\xi}=\xi_{1}-\xi_{1^{*}}$, so Assumption (A1) is also satisfied by choosing the function $V$. By doing these, (17) and (18) can be manipulated as:

$$
\begin{array}{r}
k_{1} \theta_{2}-k_{2}\left(\pi_{3}+B_{S V C 0}\right) \sin \left(\xi_{1}+\delta_{0}\right)=-\beta \sin (\tilde{\xi}) \\
k_{3}\left(-\pi_{3}+K_{c} v(\pi(\xi))=\frac{\partial \pi_{3}}{\partial \xi_{1}} \xi_{2}+\frac{\partial \pi_{3}}{\partial \xi_{2}}\left(\theta_{1} \xi_{2}-\beta \sin (\tilde{\xi})\right)\right.
\end{array}
$$

Then we define the mapping $\pi_{3}(\xi)$ which satisfies (19) first:

$$
\pi_{3}\left(\xi_{1}\right)=\frac{1}{k_{2} \sin \left(\xi_{1}+\delta_{0}\right)}\left(\beta \sin (\tilde{\xi})+k_{1} \theta_{2}\right)-B_{S V C 0}
$$

And then we design $v(\pi(\xi))$ based on (20):

$$
\begin{aligned}
& v(\pi(\xi))=\frac{1}{K_{c}} \pi_{3}+\frac{1}{k_{3} K_{c}}\left(\frac{\partial \pi_{3}}{\partial \xi_{1}} \xi_{2}+\frac{\partial \pi_{3}}{\partial \xi_{2}}\left(\theta_{1} \xi_{2}-\beta \sin (\tilde{\xi})\right)\right) \\
& =\frac{1}{K_{c}}\left(\frac{1}{k_{2} \sin \left(\xi_{1}+\delta_{0}\right)}\left(\beta \sin (\tilde{\xi})+k_{1} \theta_{2}\right)-B_{S V C 0}\right)+\frac{\xi_{2}}{k_{3} K_{c}}\left(\frac{\beta \cos (\tilde{\xi})}{k_{2} \sin \left(\xi_{1}+\delta_{0}\right)}-\frac{\left(\beta \sin (\tilde{\xi})+k_{1} \theta_{2}\right) \cos \left(\xi_{1}+\delta_{0}\right)}{k_{2} \sin ^{2}\left(\xi_{1}+\delta_{0}\right)}\right)
\end{aligned}
$$

As Step3 said, the "intermediate control law" $u=v(\pi(\xi))$ is to make the manifold $\mathcal{M}$ invariant. According to Theorem 1, the designed $u$ satisfies invariant manifold condition. Then the manifold will be defined in next step. 
(C3) Implicit manifold. The manifold $\mathcal{M}$ can be described as:

$$
\mathcal{M}:\left\{x \in \ell \in R^{n} \mid \varphi(x)=0\right\}
$$

with

$$
\varphi(x)=x_{3}-\pi_{3}\left(x_{1}\right)=x_{3}-\frac{1}{k_{2} \sin \left(x_{1}+\delta_{0}\right)}\left(\beta \sin \left(\tilde{x}_{1}\right)+k_{1} \theta_{2}\right)-B_{S V C 0}
$$

where $\tilde{x}_{1}=x_{1}-x_{1}^{*}$ is corresponding to $\tilde{\xi}=\xi_{1}-\xi_{1^{*}}$.

(C4) Manifold attractive and trajectories boundedness. In this step, the "actual control law" $u_{B}=\psi(x, z)$ is designed to guarantee that the system states' trajectories are bounded and converge to the manifold $\mathcal{M}$. The "off-the-manifold" coordinate $z$ is defined as $z=\varphi(x)$ and its dynamics is:

$$
\dot{z}=\dot{x}_{3}-\dot{\pi}_{3}\left(x_{1}\right)=k_{3}\left(-x_{3}+K_{c} \psi(x, z)\right)-\frac{\partial \pi_{3}}{\partial x_{1}} x_{2}
$$

where $u_{B}=\psi(x, z)$ is the final SVC equivalent control.

In order to ensure the boundedness of $z$ and satisfy $\lim _{t \rightarrow \infty} z(t)=0$, we let

$$
\dot{z}=-\gamma z
$$

It is apparent that in system (26), variable $z$ is bounded and will converge to zero in finite time.

By connecting equations (25) and (26) together, the control law can be got

$$
\begin{aligned}
u_{B} & =\psi(x, z)=\frac{1}{K_{c}} x_{3}+\frac{x_{2}}{K_{c} k_{3}} \frac{\partial \pi_{3}}{\partial x_{1}}-\frac{1}{K_{c} k_{3}} \gamma z \\
& =\frac{1}{K_{c}} x_{3}+\frac{x_{2}}{K_{c} k_{3}}\left(\frac{\beta \cos \left(x_{1}-x_{1}^{*}\right)}{k_{2} \sin \left(x_{1}+\delta_{0}\right)}-\frac{\left(\beta \sin \left(x_{1}-x_{1}^{*}\right)+k_{1} \theta_{2}\right) \cos \left(x_{1}+\delta_{0}\right)}{k_{2} \sin ^{2}\left(x_{1}+\delta_{0}\right)}\right)-\frac{1}{K_{c} k_{3}} \gamma\left(x_{3}\right. \\
& \left.-\frac{1}{k_{2} \sin \left(x_{1}+\delta_{0}\right)}\left(\beta \sin \left(x_{1}-x_{1}^{*}\right)+k_{1} \theta_{2}\right)-B_{S V C 0}\right)
\end{aligned}
$$

The controlled system (2) with the proposed control law (27) and "off-the-manifold" coordinate $z$ compose a closed-loop system:

$$
\left\{\begin{array}{l}
\dot{x}_{1}=x_{2} \\
\dot{x}_{2}=\theta_{1} x_{2}+k_{1} \theta_{2}-k_{2}\left(x_{3}+B_{S V C 0}\right) \delta_{\text {sin }} \\
\dot{x}_{3}=k_{3}\left(-x_{3}+K_{c} u_{B}\right) \\
\dot{z}=-\gamma z
\end{array}\right.
$$

Then we proof the boundedness of the closed-loop system states' trajectories.

From the set of the operating conditions $x=\operatorname{col}\left(x_{1}, x_{2}, x_{3}\right) \in \ell$ and $\ell=\left\{\delta-\delta_{0}, \omega-\omega_{0}, B_{S V C}-B_{S V C 0} \mid 0<\delta<\pi / 2\right\}$, we can get that state $x_{1}$ is bounded; from the definition of $z$ and $\varphi(x)$, it is implied that $x_{3}=z+\pi_{3}$, from (26), $z$ is bounded and satisfies $\lim _{t \rightarrow \infty} z(t)=0$, then $\pi_{3}$ is also bounded according to its expression, thus $x_{3}$ is bounded; then, the second equation of (28) can be rewritten as:

$$
\dot{x}_{2}=\theta_{1} x_{2}+\eta\left(x_{1}, x_{3}\right)
$$

where $\eta\left(x_{1}, x_{3}\right)=k_{1} \theta_{2}-k_{2}\left(x_{3}+B_{S V C 0}\right) \delta_{\text {sin }}$ is a bounded function which is independent of $x_{2}$, and combined with $\theta_{1}=-D / H<0$, the system presented by (29) is an exponential stable system for variable $x_{2}$, which implies that $x_{2}$ is bounded.

Finally, a proposition is given to summarize the controller design of SVC. 
Proposition 1: For the SMIB system (1) with the designed SVC nonlinear controller (27) is globally asymptotically stable at $x^{*} \in \ell$, and all the trajectories of the closed-loop system (28) are bounded.

Remark 2: In the designing of the proposed SVC control law (27), $\theta_{1}$ and $\theta_{2}$ denote $-D / H$ and $P_{m}$, respectively. While in practical $D$ and $P_{m}$ may change to some uncertain values, so on-line adaptive estimators should be designed to identify them.

\subsection{Uncertain Parameters Identification Scheme}

In this section, we design two adaptive update laws to identify the uncertain parameters $\theta_{1}$ and $\theta_{2}$ based on the adaptive sliding-mode observer for nonlinear systems. The method is based on variable structure control (VSC) theories and is completed with an application in time-varying parameters' identifications of inductor [19].

The equation includes uncertain parameters is rewritten as follows:

$$
\dot{x}_{2}=\theta_{1} x_{2}+k_{1} \theta_{2}-k_{2}\left(x_{3}+B_{S V C 0}\right) \delta_{\text {sin }}
$$

Consider the following observer based on VSC theory

$$
\dot{\hat{x}}_{2}=\hat{\theta}_{1} x_{2}+k_{1} \hat{\theta}_{2}-k_{2}\left(x_{3}+B_{S V C 0}\right) \delta_{\text {sin }}+\vartheta
$$

Where $\hat{x}_{2}, \hat{\theta}_{1}$ and $\hat{\theta}_{2}$ are the adaptive observer of $x_{2}$, estimator of $\theta_{1}$, estimator of $\theta_{2}$, respectively, and $\vartheta=-\kappa \operatorname{sign}\left(\hat{x}_{2}-x_{2}\right)$ is the input of (31), with $\kappa>0$.

Define the following new variables:

$$
\begin{aligned}
& x_{2 e}=\hat{x}_{2}-x_{2} \\
& e_{\theta 1}=\hat{\theta}_{1}-\theta_{1} \\
& e_{\theta 2}=\hat{\theta}_{2}-\theta_{2}
\end{aligned}
$$

First suppose $\hat{\theta}_{1}=\theta_{1}=\theta_{10}$ to design $\hat{\theta}_{2}$ for $\theta_{2}$, where $\theta_{10}$ is a given initial value. It follows that the dynamics of the estimation error $x_{2 e}$ is given by

$$
\dot{x}_{2 e}=k_{1} e_{\theta 2}+\vartheta=k_{1} e_{\theta 2}+\kappa \operatorname{sign}\left(\hat{x}_{2}-x_{2}\right)
$$

In order to guarantee the stability of the system (33) and the convergence of $x_{2 e}$, we suppose the following Assumption 2.

Assumption 2: In (33), the gain of the adaptive observer for $\theta_{2}$ satisfies $\kappa<-\left|k_{1} e_{\theta 2}\right|_{\max }$.

Remark 3: Under Assumption 2, we can deduce that system (33) is asymptotically stable and $x_{2 e}$ will converge to zero. This means a manifold which is described by $M_{1}=\left\{x_{2 e} \in R \mid x_{2 e}=0\right\}$ will be reached in finite time.

Then we can obtain the equivalent control $\vartheta_{e q}$ by substituting $x_{2 e}=0$ into (33).

$$
\vartheta_{e q}=-k_{1} e_{\theta 2}
$$

Remark 4: From the practical point of view, it is not possible to implement $\vartheta_{e q}$ directly because $e_{\theta 2}$ is not available. To solve this problem we use an approximation for the equivalent control $\vartheta_{e q}$ on the basis of the average control design [19]:

$$
\vartheta_{e q} \cong \frac{1}{1+\tau p} \vartheta
$$

where $\tau$ is an adjustable positive constant; $p$ is the degree of the target system, in this research, $p=2$.

Under Assumption 2 and expression (35), the parameter estimation error can be derived 
based on Remark 4.

$$
e_{\theta 2}=-\frac{1}{k_{1}} \vartheta_{e q}=-\frac{1}{k_{1}(1+2 \tau)} \vartheta
$$

Using (36), we propose the following adaptive update law:

$$
\dot{\hat{\theta}}_{2}=-K_{\theta 2} \operatorname{sign}\left(-\frac{1}{k_{1}} \vartheta_{e q}\right)=-K_{\theta 2} \operatorname{sign}\left(e_{\theta 2}\right)
$$

where $K_{\theta 2}$ is an adjustable positive coefficient. And the dynamics of the estimation error can be deduced:

$$
\dot{e}_{\theta 2}=-K_{\theta 2} \operatorname{sign}\left(-\frac{1}{k_{1}} \vartheta_{e q}\right)-\dot{\theta}_{2}
$$

Then design the estimator $\hat{\theta}_{1}$ for $\theta_{1}$ while the value of $\theta_{2}$ is fixed to $\hat{\theta}_{2}$. The procedure is similar with the designing of $\hat{\theta}_{2}$, for length limitation, the detailed process is omitted. We just give the designing result:

$$
\dot{\hat{\theta}}_{1}=-K_{\theta 1} \operatorname{sign}\left(-\frac{1}{x_{2}} \vartheta_{e q}\right)=-K_{\theta 1} \operatorname{sign}\left(e_{\theta 1}\right)
$$

The main result of the adaptive estimators designing can be summarized by the following theorem:

Theorem 2: The SVC nonlinear system is expressed by (2) and the adaptive update laws are expressed by (37) and (39). If all the trajectories of the system (2) are continuous and bounded, then the estimated values given by the integrators of (37) and (39) converge to their corresponding true values $\theta_{2}$ and $\theta_{1}$ in finite time.

Proof: Given the following Lyapunov function is constructed:

$$
V=\frac{1}{2} e_{\theta 2}{ }^{T} e_{\theta 2}
$$

And taking its time-derivative along the solution of (37) and (39), we obtain:

$$
\begin{aligned}
\dot{\boldsymbol{V}} & =\boldsymbol{e}_{\theta 2}{ }^{\boldsymbol{T}} \dot{\boldsymbol{e}}_{\theta 2} \\
& =-\boldsymbol{e}_{\theta 2}{ }^{\boldsymbol{T}} \boldsymbol{K}_{\theta 2} \operatorname{sign}\left(\boldsymbol{e}_{\theta 2}\right)-\boldsymbol{e}_{\theta 2}{ }^{\boldsymbol{T}} \dot{\theta}_{2}-\left|\boldsymbol{e}_{\theta 2}{ }^{T}\right|\left(\boldsymbol{K}_{\theta 2}\left|\operatorname{sign}\left(\boldsymbol{e}_{\theta 2}\right)\right|\right)-\left|\boldsymbol{e}_{\theta 2}{ }^{\boldsymbol{T}}\right| \sigma_{2} \\
& =-\left|\boldsymbol{e}_{\theta 2}{ }^{\boldsymbol{T}}\right|\left(\left(\boldsymbol{K}_{\theta 2}\left|\operatorname{sign}\left(\boldsymbol{e}_{\theta 2}\right)\right|\right)+\sigma_{2}\right)
\end{aligned}
$$

where $K_{\theta 2}>0, \sigma_{2} \geq 0$ and then we have $\dot{V} \leq 0$. It can be summarized that the differential of the Lyapunov function (41) keep negative definite for any value of $e_{\theta 2}$, which means that the estimation error $e_{\theta 2}$ will converge to zero by recalling Lyapunov stability theory. That is to say, the proposed adaptive update law (37) can identify the true value of $\theta_{2}$ through designing $\hat{\theta}_{2}$. The proof of the convergence of the adaptive update law $\dot{\hat{\theta}}_{1}$ is skipped for it has the similar certification process.

\section{Simulation Results and Discussion}

Simulation of the closed-loop system (28), with the control law (27) and adaptive update laws (37) and (39) are carried out by using Matlab/Simulink tool. The SMIB system parameters for simulation are a s follow s : $H=5.9 \mathrm{~s} ; V_{\mathrm{s}}=1.0 \mathrm{pu} ; \omega_{0}=314.2 \mathrm{rad} / \mathrm{s} ; T_{c}=0.02 \mathrm{~s} ; K_{c}=1 ; E_{q}{ }^{\prime}=1.08 \mathrm{pu}$; $X_{1}=0.84 p u ; X_{2}=0.52 p u ; B_{L}-B_{C}=0.3 p u$. The initial operation equilibrium point is $\left[\begin{array}{lll}\delta_{0} & \omega_{0} & B_{S V C O}\end{array}\right]^{T}=\left[\begin{array}{lll}0 & 0 & 1.1\end{array}\right]^{T}$. The initial values of the uncertain parameters 
are $\hat{\theta}_{1}(0)=0$ and $\hat{\theta}_{2}(0)=1.66$. We investigate the transient responses of the controlled system when a severe permanent fault appears on the generator shift, this is tantamount to a step change in $P_{m}$. The simulations are started with the following designed parameters: $\beta=5 ; \gamma=15 ; K_{\theta 1}=0.00015 ; K_{\theta 2}=0.00015$. For the sake of comparison, the classical adaptive backstepping method based controller is also fulfilled for SMIB system under the same operating conditions.

Figure 3 shows the trajectories of the system state variables $x_{1}, x_{2}$ and $x_{3}$ under the proposed method and the classical adaptive backstepping method, respectively. From these three subfigures, the curves that imposed by the proposed method respond fast after the fault occurred and converge to the initial stable equilibrium speedily. This means that the generator rotor angle and its angular speed and the equivalence susceptance of SVC system are regulated to their initial stable value after the fault occurs by the proposed method. And the trajectories reach to the new stable with slighter oscillations compared by the trajectories that imposed by the classical adaptive backstepping method. These results imply that the transient stability of the SMIB system is improved by the proposed SVC controller when severe fault occurs.
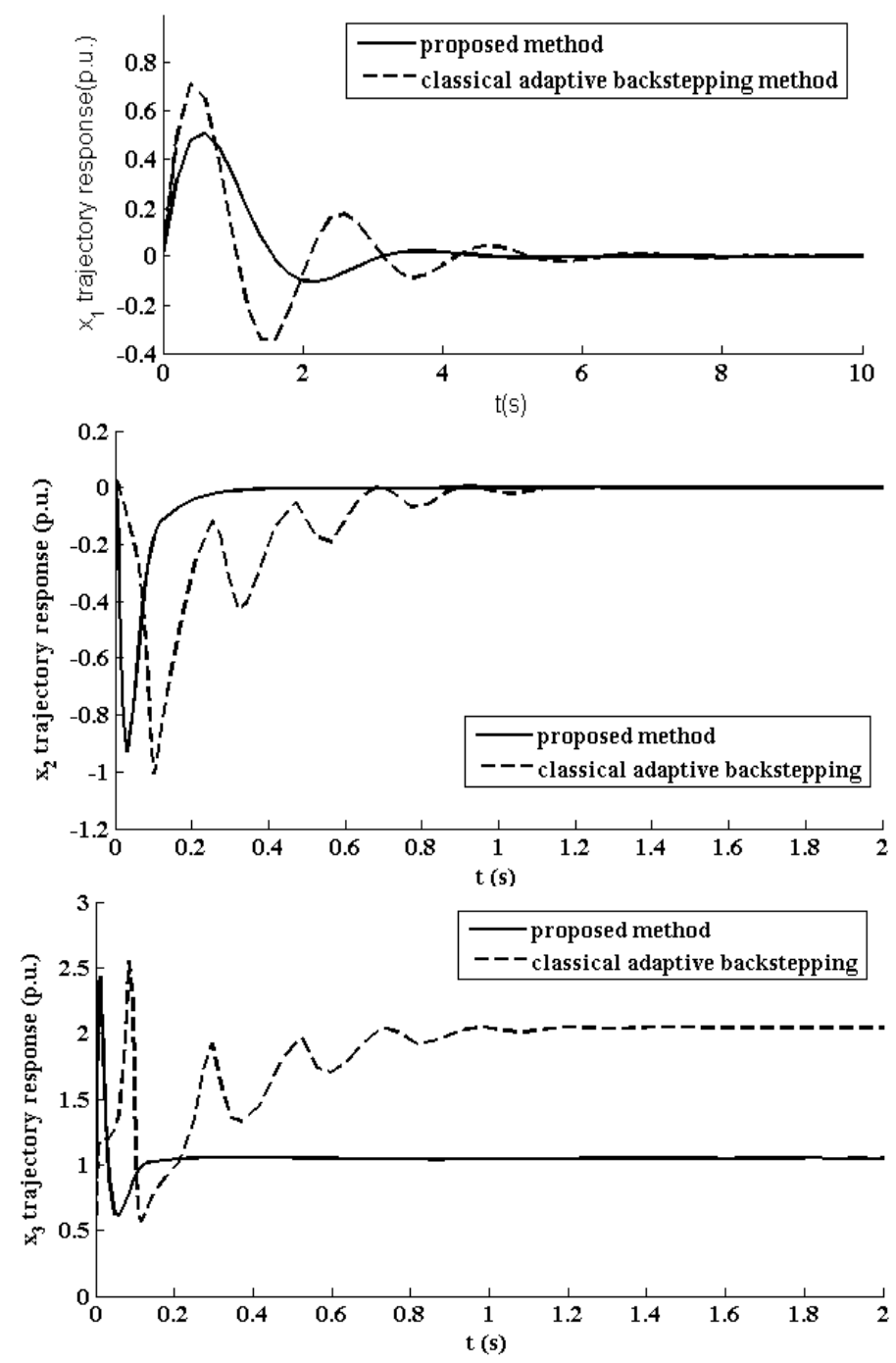

Figure 3. Transient Trajectories of States $x_{1}, x_{2}$ and $x_{3}$ 
Figure 4 shows the trajectories of $x_{1}$ and $x_{2}$ in different $\gamma$ and the other parameters are fixed to the initial values. The value of $\gamma$ are selected as 15 and 30. From the two subfigures, when $\gamma=15$ the response trajectories of states $x_{1}$ and $x_{2}$ converge faster than that of $\gamma=30$, and present slighter oscillations. This means that the smaller the value of $\gamma$ is selected, the more excellent transient responses of the system can be achieved.
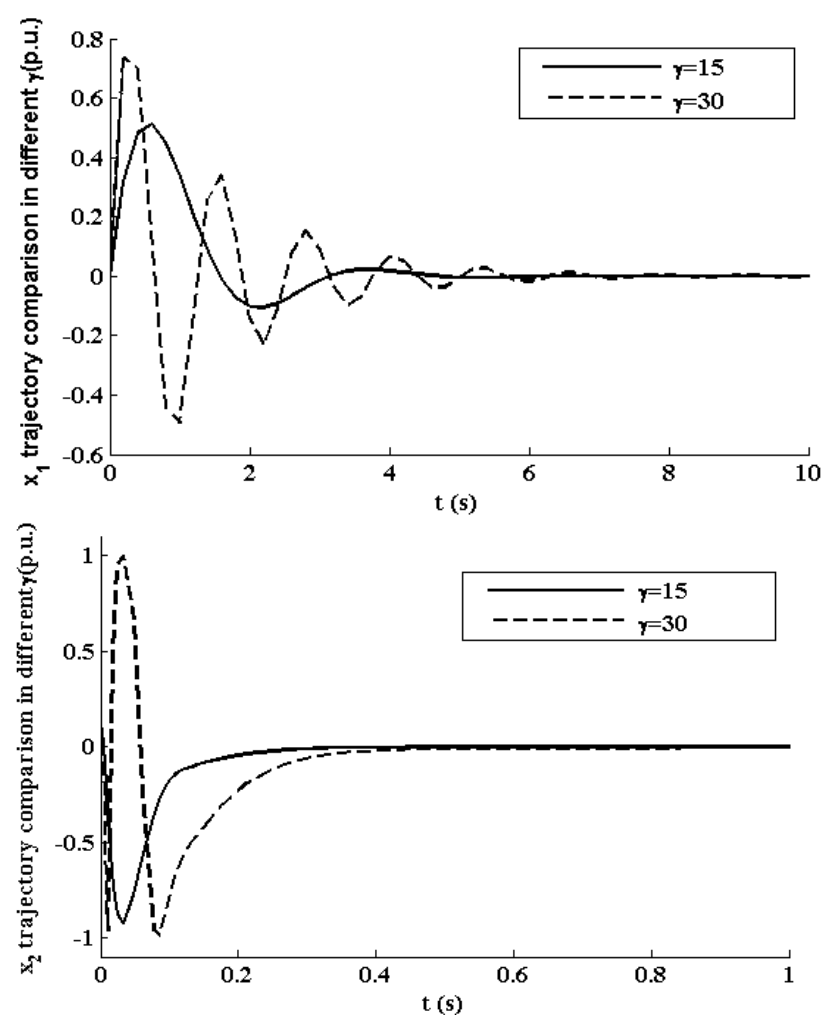

Figure 4. Transient Trajectories of States $x_{1}$ and $x_{2}$ in Different $\gamma$

Figure 5 presents the terminal voltage of SVC that expressed by $V_{t}$ under different values of $\gamma$. It can be seen that $V_{t}$ can be regulated to its initial stable value fast after the fault occurs. And the response of $V_{t}$ is markedly affected by the value of $\gamma$. The smaller the value of $\gamma$ is, the slighter and smaller oscillation amplitude the response trajectory of $V_{t}$ will has.

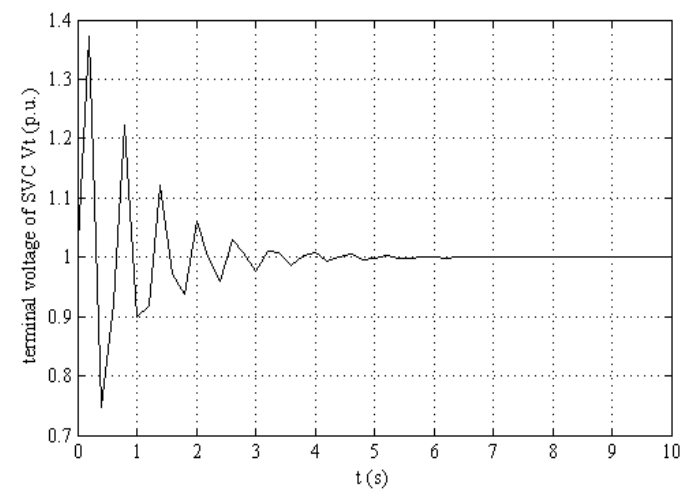




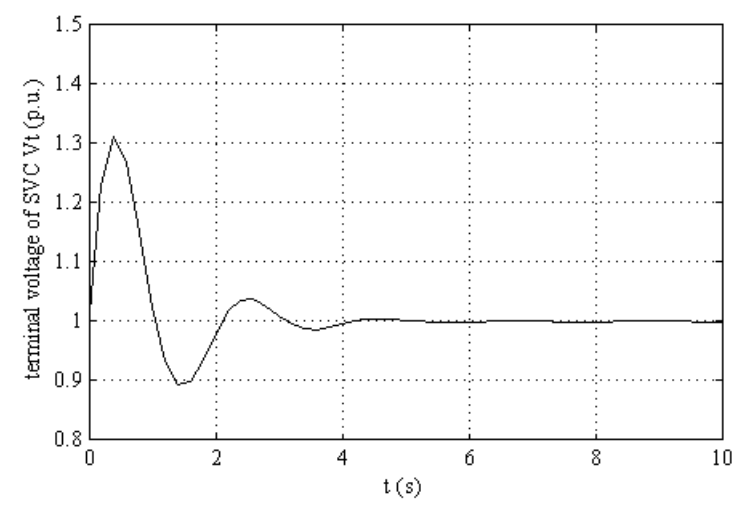

Figure 5. Terminal Voltage of SVC when $\gamma=15$ and $\gamma=30$

We fix the value of $\gamma$ as 15 , and simulate the identification results of the uncertain parameters $\theta_{1}$ and $\theta_{2}$ when they are changed to a new value. The results are shown in Figure 6. From the response curves of $\hat{\theta}_{1}$ and $\hat{\theta}_{2}$, we can see that the estimators can follow the change of $\theta_{1}$ and $\theta_{2}$, and converge to their true values finally. It can also be interpreted that the estimation errors converge to zero, which means that the proposed adaptive update laws (37) and (39) can effectively identify the uncertain parameters.
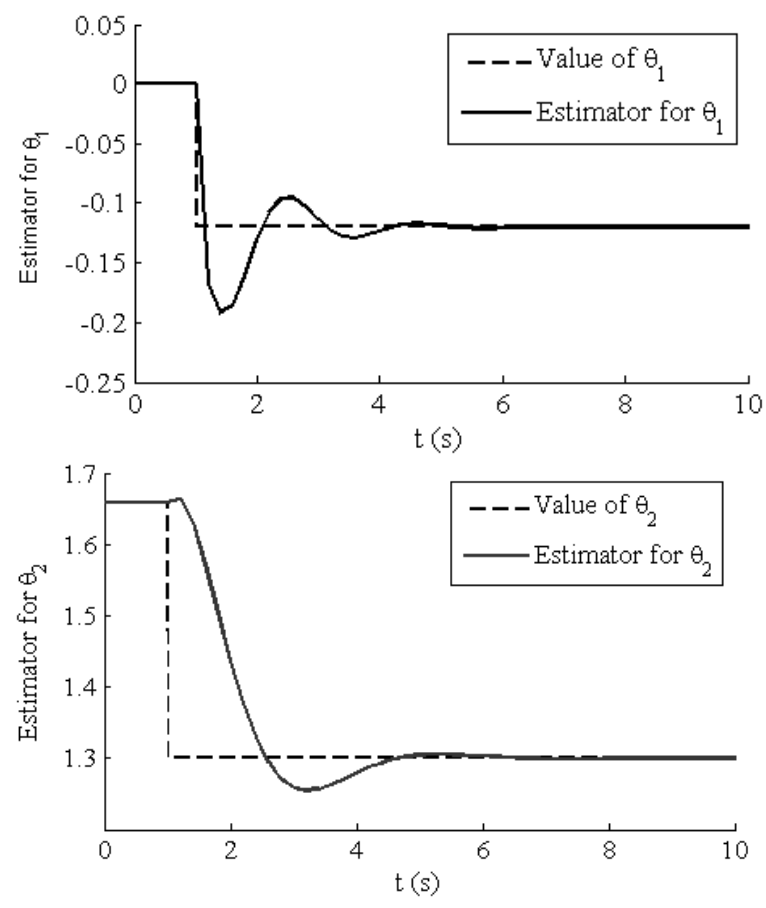

Figure 6. Responses of the Estimators of $\theta_{1}$ and $\theta_{2}$

\section{Conclusions}

In this paper, an adaptive nonlinear SVC controller is designed on the basis of I\&I methodology to improve the transient stability of a SMIB system. In the SVC controller design, the uncertain damping coefficient, mechanical power, generator rotor angle and line impedance are taken into consideration. The transient stability of the controlled system is guaranteed through defining a second-order stable target system and immersing the third-order controlled system into it based on I\&I methodology. The proposed controller is 
made adaptive by designing adaptive update laws to identify the uncertain parameters on the basis of adaptive sliding-mode observer. The simulation results show that the transient stability of the SMIB system is improved than the classical adaptive backstepping method, and the proposed adaptive update laws can effectively identify the variable uncertain parameters. The prospective working will focus on the robustness of the adaptive control system, which will make the system transient performance is not affected by the uncertain disturbances.

\section{Acknowledgement}

The authors would thank the editors and reviewers for their useful comments to improve the quality of the manuscript. The project is supported by National Natural Science Foundation of China (NSFC: 51177126), Scientific Research Program Funded by Shaanxi Provincial Education Department (14JK1306) and Xi 'an Polytechnic University Doctoral Research Start Funded Projects (BS1337).

\section{References}

[1] A.E. Hammad "Analysis of Power System Stability Enhancement By Static Var Compensators", IEEE Transactions on Power System, vol. 1, no. 4, (1986), pp. 222-227.

[2] J.S.K Leung, D.J. Hill and Y.X. Ni, "Global Power System Control Using Generator Excitation, PSS, FACTS Devices and Capacitor Switching”, International Journal of Electrical Power \& Energy Systems, vol. 27, no.5, (2005), pp. 448-464.

[3] E. Z. Zhou, "Application of Static Var Compensators to Increase Power System Damping", IEEE Transactions on Power System, vol. 8, no.2, (1993), pp. 205-216.

[4] P. Wang and W. W. Sun, "Adaptive Ho Control for Nonlinear Hamiltonian Systems with Time Delay and Parametric Uncertainties", International Journal of Automation and Control, vol. 11, no.4, (2014), pp. 368-376.

[5] Y. Wang, H. Chen and R. Zhou, "A Nonlinear Controller Design for SVC to Improve Power System Voltage Stability”, IEEE Transactions on Electrical power and energy systems, vol. 22, no. 7, (2000), pp. 463-470.

[6] Q.H. Wu and L. Jiang, "Nonlinear Adaptive Co-ordinated Control of Multimachine Power Systems", Transactions of the Institute of Measurement and Control, vol. 24, no.3, (2002), pp. 195-213.

[7] S. Robak, "Robust SVC Controller Design and Analysis For Uncertain Power Systems", Control Engineering Practice, vol. 17, no.11, (2009), pp. 1280-1290.

[8] A. Karimi, Muhammad, A. Choudhry and A. Feliachi, "Coordinated Backstepping Controls for Power System Stability Enhancement", Proceedings of the North American Power Symposium, Washington, USA, (2008), pp. 1-8.

[9] R. Yan, Z.Y. Dong and T.K. Saha, "Power System Transient Stability Enhancement With An Adaptive Control Scheme Using Backstepping Design", Proceedings of the IEEE Power and Energy Society General Meeting, Tampa, FL, USA, (2007), pp. 1025-1032.

[10] L.Y. Sun, S.C. Tong and Y. Liu, "Adaptive Backstepping Sliding Mode $H \infty$ Control of Static Var Compensator", IEEE Transactions on Control System Technology, vol.19, no. 5, (2011), pp. 1133-1140.

[11] C.Y. Wen, Y. Zhang and Y.C. Soh, "Robustness of An Adaptive Backstepping Controller without Modification", Systems \& Control Letters, vol. 36, no.2, (1999), pp. 87-100.

[12] A. Astolfi and R. Ortega, "Immersion and Invariance: A New Tool for Stabilization and Adaptive Control of Nonlinear Systems", IEEE Transactions on Automatic Control, vol. 48, no.4, (2003), pp. 590-606.

[13] D. Karagiannis and A. Astolfi, "Nonlinear Adaptive Control of Systems in Feedback Form: An Alternative to Adaptive Backstepping", Systems \& Control Letters, vol. 57, no. 9, (2008), pp. 238-289.

[14] W. Dib, G. Kenné and F. Lamnabhi, "An Application of Immersion and Invariance To Transient Stability and Voltage Regulation of Power Systems With Uncertain Mechanical Power", Proceedings of the Joint 48th IEEE Conference on Decision and Control and 28th Chinese Control Conference, Shanghai,China, (2009), pp. 3337-3342.

[15] W. Dib and R. Ortega, "Improving Transient Stability of Multi-machine Power Systems: Synchronization via Immersion of A Pendulum System", Proceedings of the American Control Conference, San Francisco, California, USA, (2011), pp. 1408-1413.

[16] F. Jun, Zhao Jun and G. M. Dimirovsk, "Robust Control of SVC: A New Adaptive Backstepping Method", Proceedings of the CESS, vol. 26, no. 10, (2006), pp. 7-11. 
[17] L. Cong, Y. Wang and D.J. Hill, "Co-ordinated Control Design of Generator Excitation and SVC for Transient Stability and Voltage Regulation Enhancement of Multi-machine Power Systems", International Journal of Robust and Nonlinear Control, vol. 14, no.9, (2004), pp. 789-805.

[18] T. Ahmed-Ali, F. Floret and F. Lamnabhi-Lagarrigue, "Robust Identification and Control with Time-varying Parameter Perturbations", Proceedings of the American Control Conference, Denver, Colorado, USA, (2003), pp. 1908-1913.

[19] G. Kenné, T. Ahmed-Ali. and Lamnabhi-Lagarrigue, "Real-time Speed and Flux Adaptive Control of Induction Motors Using Unknown Time-varying Rotor Resistance And Load Torque", IEEE Transactions on Energy Conversion, vol. 24, no.2, (2009), pp. 375-387.

\section{Authors}

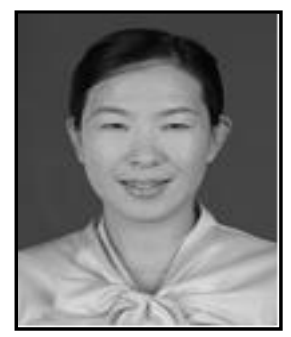

Lei Zhang, She received her B.Sc. and M.Sc. degrees in Control Theory and Control Engineering from Xi'an Polytechnic University, China, in 2004 and 2007, respectively, and Ph.D. degree in Control Science and Engineering from Xi' an Jiaotong University in 2013. Her research interest includes nonlinear control theory, power system transient stability, robust control theory and its applications in industrial engineering. E-mail: carol1208@163.com 